\title{
Epithelial-mesenchymal transition sensitizes breast cancer cells to cell death via the
}

\section{fungus-derived sesterterpenoid ophiobolin A}

Keighley N. Reisenauer ${ }^{1}$, Yongfeng Tao ${ }^{2}$, Shuxuan Song ${ }^{1}$, Saawan D. Patel ${ }^{1}$, Alec Ingros ${ }^{1}$, Peter Sheesley ${ }^{1}$, Marco Masi ${ }^{3}$, Angela Boari ${ }^{4}$, Antonio Evidente ${ }^{3}$, Alexander V. Kornienko ${ }^{5}$,

Daniel Romo ${ }^{2}$, Joseph Taube ${ }^{1 \#}$

\section{Affiliations}

${ }^{1}$ Department of Biology, Baylor University, Waco, TX, USA

${ }^{2}$ Department of Chemistry and Biochemistry, Baylor University, Waco, TX, USA

${ }^{3}$ Department of Chemical Sciences, University of Naples Federico II, Complesso Universitario Monte Sant'Angelo, Naples, Italy

${ }^{4}$ Institute of Sciences and Food Production, CNR, Bari, Italy

${ }^{5}$ Department of Chemistry and Biochemistry, Texas State University, San Marcos, TX, USA

\#Corresponding author: Joseph Taube, Joseph Taube@baylor.edu 


\section{$\underline{\text { Abstract }}$}

The epithelial-mesenchymal transition (EMT) imparts properties of cancer stem-like cells, including resistance to frequently used chemotherapy, necessitating the identification of molecules that induce cell death specifically in stem-like cells with EMT properties. Herein, we demonstrate that breast cancer cells enriched for EMT features are more sensitive to cytotoxicity induced by ophiobolin A (OpA), a sesterterpenoid natural product. Using a model of experimentally induced EMT in human mammary epithelial (HMLE) cells, we show that EMT is both necessary and sufficient for OpA sensitivity. Moreover, prolonged, sub-cytotoxic exposure to OpA is sufficient to reduce migration, sphere formation, and resistance to doxorubicin. OpA is well-tolerated in mice and treatment with OpA alone reduces tumor burden. These data identify a driver of EMT-driven cytotoxicity with significant potential for use either in combination with standard chemotherapy or for tumors enriched for EMT features.

\section{$\underline{\text { Introduction }}$}

Breast cancer patients who have triple-negative breast cancer (TNBC) face poor prognoses driven by high rates of metastasis and early recurrence ${ }^{1-6}$. TNBC is characterized as histologically negative for estrogen receptor (ER), progesterone receptor (PR), and amplified human epidermal growth factor receptor-2 (HER2), preventing the use of hormone- or HER2targeted therapies. Instead, treatment with anthracyclinedoxorubicin) and/or taxanes is only capable of providing 5-year survival in about half of TNBC patients ${ }^{7-10}$.

TNBC is comprised of mostly basal-like and claudin-low intrinsic subtypes, both of which have been characterized as enriched with cancer stem-like cells ${ }^{11-13}$. Cancer stem-like cells (CSCs) are defined by their ability to re-initiate tumor growth upon transplantation and are hypothesized to fuel metastasis and primary tumor recurrence, resulting in an overall decrease 
in survival ${ }^{14-17}$. To improve TNBC patient outcomes, novel and specific approaches targeted at CSCs are needed.

One proposed mechanism driving the emergence of CSC-like cells is the epithelialmesenchymal transition, EMT ${ }^{18,19}$. EMT is a trans-differentiation process characterized by spindle-like morphology, loss of apical-basal polarity, increased motility, and a tolerance to anoikis. These phenotypic shifts are driven by gene expression changes mediated by transcription factors Snail (SNA/1), Twist (TWIST1), and ZEB1, effects of which include upregulation of vimentin and $\mathrm{N}$-cadherin, and downregulation of epithelial markers $\mathrm{E}$-cadherin and miR-200c ${ }^{20-26}$.

Cells that have undergone an EMT typically acquire CSC properties including decreased sensitivity to conventional chemotherapies used to treat TNBC. This chemoresistance is driven by drug efflux pumps, enhanced DNA repair capacity, mesenchymal-like properties, and epigenetic changes ${ }^{16,27-32}$. There are currently no approved therapies that specifically target CSCs. A leading pre-clinical compound is salinomycin, reported to decrease the subpopulation of CSCs, tumor initiating capability, and chemoresistance, with negligible side effects ${ }^{33}$. Other naturally occurring compounds such as curcumin, and quercetin have been reported to reduce the effects of EMT by inhibiting key proteins associated with migration (Snail, MMP-2/9), anoikis tolerance (Bcl-2), cell-to-cell adhesion (N-cadherin), and signaling cascades (JAK/STAT, ERK) ${ }^{34-37}$.

Ophiobolin A (OpA) is a natural product produced from fungi in the genera Aspergillus, Bipolaris, Cephalosporium, Cochliobolus, and Drechslera ${ }^{38}$. This sesterterpenoid (25-carbons) is a secondary metabolite that has long been studied for its phytotoxic effects in a variety of plants and has begun to be evaluated as a cytotoxic compound ${ }^{38}$. Recently published cell 
culture-based experiments describe a role for OpA in motility inhibition ${ }^{39}$, membrane depolarization ${ }^{40-43}$, roles in inflammation ${ }^{44}$, and reduction in stemness ${ }^{45}$. In vivo data demonstrate that OpA is tolerated in mice and is effective against an orthotopic model of glioblastoma $^{40,46,47}$. Herein, we investigated the applicability of OpA on EMT-enriched breast cancer and found that experimentally induced EMT enhances the susceptibility of mammary epithelial cells to OpA-induced cell death. Furthermore, breast cancer cell lines treated with OpA experience loss of both stemness and migratory attributes, demonstrating that OpA induces selective cytotoxicity in cells that have undergone EMT. Additionally, OpA is effective in reducing tumor burden in mice with orthotopic, EMT-positive, mammary tumors, highlighting the potential of EMT-targeted cancer treatment.

\section{$\underline{\text { Results }}$}

\section{Mammary epithelial cells that have undergone EMT are more sensitive to OpA}

Given the previously published link between OpA and CSC-targeted activity ${ }^{45}$, we investigated a potential link between OpA (Fig. 1A) and EMT using an experimental model of EMT induction. Immortalized human mammary epithelial (HMLE) cells have an epithelial morphology and express E-cadherin. We used HMLE cells, as well as HMLE cells transformed with the Ras oncoprotein (HMLER) that are induced to undergo EMT through lentiviral transduction of viruses driving expression of the EMT-inducing transcript factor Twist, ${ }^{26,48}$ resulting in the acquisition of a mesenchymal morphology (Fig 1A) and protein expression (Fig. 1B). We measured the Twist-induced selective sensitivity to molecules shown to inhibit CSC properties including salinomycin ${ }^{49}$, ophiobolin $A^{45}$, curcumin ${ }^{50}$, genistein ${ }^{51}$, and disulfiram ${ }^{52}$.

Only two such molecules demonstrated selectivity towards EMT-positive cells, salinomycin and 
ophiobolin A, and only OpA also demonstrated sub-micromolar cytotoxic activity (Fig. 1C).

Furthermore, induction of EMT through expression of Twist or through another EMT-TF, Snail

${ }^{53}$, in either HMLE or HMLER cells increased sensitivity to OpA-driven cytotoxicity (Fig. 1D).

Indeed, the EMT decreased the $\mathrm{IC}_{50}$ value from a mean of 137-147 nM for epithelial cells to a mean of 85-91 nM for mesenchymal cells (Fig. 1E). These results stand in stark contrast to EMT-driven resistance to many commonly used chemotherapeutic drugs including doxorubicin, paclitaxel ${ }^{49}$, and staurosporine (Fig. 1F).

\section{miR-200c suppression is necessary for sensitivity to OpA}

Because we observed that OpA selectively impacts cells that have undergone EMT, we next evaluated whether reversing the EMT status of these cells would be sufficient to restore OpA sensitivity. To do this, we introduced a ZEB1-targeting microRNA into HMLE-Twist and HMLER-Twist cells. miR-200c expression has been shown to be sufficient to reverse EMT ${ }^{54}$. We verified over-expression of miR-200c driven by transduction with a lentiviral vector (Fig. 2A). We next measured sensitivity to OpA and found that induction of miR-200c partially restored resistance to $\mathrm{OpA}(\mathrm{Fig} 2 \mathrm{~B})$. This indicates that expression modulation of the EMT state impacts sensitivity to OpA.

\section{Persistent treatment with OpA alters cellular phenotypes}

Engaging the EMT program can confer stemness properties in cancer cells (Mani et al., 2007; Morel et al., 2008). To examine the effect of OpA on breast cancer cells, we measured the cytotoxic activity on the ER-positive, CSC-poor, epithelial-like MCF7 and triple-negative, CSCrich, mesenchymal-like MDA-MB-231 cell lines. While both cell lines were highly responsive to OpA, the MDA-MB-231 cells displayed significantly greater cell death at an $80 \mathrm{nM}$ dose (Fig. 3A). To evaluate the impact of sub-cytotoxic doses of OpA on EMT and CSC phenotypes, we 
performed experiments on CSC-rich MDA-MB-231 cells using continuous, multi-day treatment of $30 \mathrm{nM}$ OpA or $30 \mathrm{nM}$ deoxy-OpA, as a negative control (Fig. 3B-black arrow). Continuous treatment with a sub-cytotoxic doses of OpA, but not 3-deoxy OpA triggered modest changes in cell morphology toward a more compact and cobblestone-like appearance, characteristic of epithelial cells (Fig. 3C). EMT is necessary for the migratory capacity of MDA-MB-231 cells. To ascertain if OpA inhibited migration we performed a wound healing assay. Consistent with an effect on EMT properties, cells pre-treated with sub-cytotoxic doses of OpA, but not 3-deoxyOpA, failed to migrate in response to a scratch wound (Fig. 3D,E). We next tested CSCcharacteristic anchorage-independent growth using a mammosphere assay. Consistent with an effect on CSC properties, we observed that pre-treatment of MDA-MB-231 cells with OpA reduced sphere formation (Fig 3F). In summary, persistent treatment of a CSC-rich breast cancer cell line with OpA diminishes sphere formation and migratory properties associates with CSC and EMT.

\section{OpA treatment increases sensitivity to chemotherapy}

EMT-promoted stemness drives resistance to commonly used chemotherapies. One approach to overcoming this problem is to consider dual-treatment therapies that combine CSC-targeting compounds with conventional drugs. To examine the combinatorial impact of OpA treatment we co-treated MDA-MB-231 cells with a dilution series of OpA and either doxorubicin or paclitaxel. Co-treatment with as little as $12.5 \mathrm{nM}$ OpA enhanced the cytotoxic response from doxorubicin (Fig. 4A), while 50 nM OpA enhanced the cytotoxic response from paclitaxel (Fig. 4B). Notably, addition of $50 \mathrm{nM}$ OpA was sufficient to maintain cytotoxic activity despite a 25fold reduction in the dose of doxorubicin (Fig. 4A-orange bar) and a 5-fold reduction in the dose of paclitaxel (Fig. 4B-orange bar). Indeed, when analyzed using Combenefit, these dose 
combinations tended toward synergistic effects (Fig. 4C,D). Combination treatment using 3deoxy-OpA did not result in altered activity (Fig. 4E,F) The capacity of OpA to act in concert with clinically useful chemotherapeutic agents indicates that co-treatment may be useful to more effectively treat breast cancer.

\section{OpA is tolerated in vivo and suppresses Twist-expressing tumor growth}

We next assessed whether OpA treatment alone is sufficient to reduce tumor growth in mice. In order to gauge the impact of OpA on tumor growth, immunocompromised mice were orthotopically injected with Ras-transformed HMLE cells expressing the Twist transcription factor (HMLER-Twist) to induce EMT. Following the emergence of palpable tumors, mice were randomly assigned to either the control (DMSO diluted into saline) or OpA treatment groups. Thrice weekly injections for 3 weeks consisting of $10 \mathrm{mg} / \mathrm{kg}$ of OpA were not well tolerated as mice exhibited weight loss greater than $20 \%$ of initial body weight and two adverse outcomes were recorded prior to the final dose (Fig. 5A). However, a dose of $5 \mathrm{mg} / \mathrm{kg}$ was better tolerated with final weight loss less than $15 \%$ and one adverse outcome while a dose of 2.5 $\mathrm{mg} / \mathrm{kg}$ had no statistically significant impact on body weight (Fig. 5A). A dose of $5 \mathrm{mg} / \mathrm{kg}$ of OpA was sufficient to significantly reduce the endpoint tumor volume of HMLER-Twist tumors (Fig. 5B).

\section{Discussion}

Currently, conventional chemotherapeutic drugs are able to elicit high response rates in about half of TNBC patients; however, the remaining patients eventually develop progressive disease $^{2}$, with some even experiencing more aggressive and CSC-rich tumors after therapy ${ }^{14,55}$. Identification of molecules that mediate sensitivity to CSC-rich cell populations will 
facilitate the development of novel therapies and may improve responses to currently available therapies.

While several other natural products have been linked to CSC-targeting ${ }^{34,35,49,56-61}$, our work highlights a natural product that selectively kills breast CSCs exhibiting EMT features. We demonstrate selective and necessary sensitivity to OpA in cells that have undergone EMT. Further, we show a reduction of EMT phenotypes such a migration and morphology, as well as reduction in sphere-forming capacity in a TNBC cell model. Extending OpA's efficacy in reducing CSC-related properties, our data suggest increased sensitivity to conventional chemotherapeutics doxorubicin and paclitaxel when co-treated with OpA. Finally, we evaluate the efficacy of OpA in vivo and show a high level of tolerance to OpA, as well as volume reduction in an EMT-positive, primary tumor.

Evolution-driven selection of natural products imparts biological activities useful for disease treatment and which may not be mimicked by selective kinase inhibitors. Other successful natural products that have driven cancer therapies include taxol, vinblastin, anthracyclines, daunomycin and doxorubicin ${ }^{62}$. Many studies ${ }^{40,42,43,47,63-66}$ have evaluated one such natural product, OpA, in cancer settings, predominantly using in vitro models, and, similar to our present study, these studies report $\mathrm{IC}_{50}$ values in the low nanomolar range. Our work is one of the first to evaluate OpA in vivo and is the first to describe the impact of EMT on OpA sensitivity. By focusing on the effects on EMT and stemness phenotypes, this work opens the door for the discovery of essential molecular pathways and for the investigation of OpA derivatives as a future cancer treatment. 


\section{Materials and Methods}

\section{Cell Lines}

MCF7 were received from ATCC. MDA-MB-231, Hs578T, HMLE, HMLER, HMLE Twist, and HMLER Twist were kindly gifted from Dr. Sendurai Mani (MD Anderson Cancer Center). Breast cancer cells were cultured in Dulbecco's Modified Eagle's Medium (DMEM) (Corning Inc., Kennebuck, ME, USA) supplemented with 10\% fetal bovine serum (FBS) (Equitech-Bio Inc., Kerrville, Texas, USA) and 1X antibiotics (Penicillin/Streptomycin, Lonza, Basel, Switzerland). Immortalized human mammary epithelial cells (HMLE) and derivatives were maintained as in Elenbaas et. al ${ }^{67}$. Cell lines were tested monthly for mycoplasma and validated via STR testing. Culture conditions were $37^{\circ} \mathrm{C}, 5 \% \mathrm{CO}_{2}$.

\section{Reagents}

OpA was produced by fermentation of the fungus Drechslera gigantea. It was extracted from the fungal culture filtrates, purified, crystallized and identified by ${ }^{1} \mathrm{H}$ NMR and ESI MS as previously reported ${ }^{68}$. The purity of OpA was $>98 \%$ as ascertained by ${ }^{1} \mathrm{H}$ NMR and HPLC analyses.

3-Deoxy OpA was synthesized from ophiobolin $\mathrm{I}^{69,70}$ which was also obtained through fermentation as previously reported ${ }^{68}$. A two-step synthetic sequence involving conjugate reduction of the enone which proceeded with high diastereoselectivity (>19:1 by $600 \mathrm{MHz}{ }^{1} \mathrm{H}$ NMR) followed by a Ru(IV)-mediated oxidation of the primary alcohol to the aldehyde delivered 3-deoxy OpA. It should be noted that the methyl group at C3 is epimeric with respect to the C3-methyl group in OpA. However, the importance of the C3-hydroxy group and/or the stereochemistry of this methyl group was verified through studies described below and 3- 
deoxy OpA served as a negative control. Further details are provided in Supplemental Figure 1.

\section{Viability}

Cells were plated with 2,000 cells per well in a 96 -well plate and allowed to adhere overnight. Compounds, suspended in DMSO and diluted into PBS, or vehicle were added to the culture medium and incubated for 72 hours at $37{ }^{\circ} \mathrm{C}, 5 \% \mathrm{CO}_{2}$. Following manufacturer suggested protocol, $20 \mu \mathrm{L}$ CellTiter $96 \AA \mathrm{AQ}_{\text {ueous }}$ One Solution Cell Proliferation Assay (MTS; Promega, Madison, WI, USA) was added and incubated $1-4$ hours at $37^{\circ} \mathrm{C}, 5 \% \mathrm{CO}_{2}$. Absorbance was measured at $490 \mathrm{~nm}$ using a 96-well plate reader (Fisher Scientific, Hampton, NH, USA).

\section{RNA extraction and detection}

Cells were lysed in the presence of Trizol® Reagent (Thermo Scientific, Waltham, MA, USA) and total RNA extracted following manufacturer protocol recommendations. Relative quantification of the mRNA levels was performed using the comparative Ct method with Taqman assays for U6 as the reference gene for microRNA analysis and SYBR Green assays GAPDH as the reference gene for mRNA analysis, and with the formula $2^{-\Delta \Delta C t}$ (Applied Biosystems, Foster City, CA, USA; Thermo Scientific). All quantitative reverse transcriptionPCR (RT-PCR) experiments were run in triplicate and a mean value was used for the determination of mRNA levels.

\section{Western blotting and antibodies}

Cells were lysed in the presence of 100 microliters radio-immunoprecipitation (RIPA) buffer containing protease inhibitors (Alfa Aesar, Stoughton, MA, USA) on ice. Protein was quantified using the Bradford Assay (BioRad, Hercules, CA, USA). Twenty micrograms of total protein 
from each sample was resolved on a 4\%-12\% SDS-PAGE gel and transferred to PVDF membranes. Sister blots were then probed with antibodies including anti-E-cadherin (Cell Signaling, Danvers, MA, USA), anti-Fibronectin (Sigma-Aldrich, St. Louis, MO, USA), antivimentin (Protein Technologies, Tucson, AZ, USA), anti-N-cadherin (Cell Signaling), anti-ZEB1 (Santa Cruz Biotechnologies, Dallas, TX, USA), or anti- $\beta$-actin (BD Biosciences, San Jose, CA) antibody. Chemiluminescent signals were detected with ECL ${ }^{\mathrm{TM}}$ prime (Thermo Fisher Scientific) using the Biorad ChemiDoc system. If necessary, blots were stripped with ECL Stripping Buffer (Li-Cor, Lincoln, NB, USA) following manufacturer protocol. Bands were quantified using ImageJ.

\section{Mammosphere Assay}

Cells were harvested according to standard protocol and were suspended in serum-free mammary epithelial growth medium (MEGM) supplemented with 1\% methyl cellulose, 20 $\mathrm{ng} / \mathrm{mL}$ FGF, $10 \mathrm{ng} / \mathrm{mL}$ EGF, and $4 \mu \mathrm{g} / \mathrm{mL}$ heparin. Cells were plated in 4 replicates in a flatbottom ultra-low attachment 96-well plate (Corning) and allowed to grow at $37{ }^{\circ} \mathrm{C}, 5 \% \mathrm{CO}_{2}$ for 10-14 days and were monitored microscopically to ensure that they did not become confluent during the experiment. $100 \mu \mathrm{L}$ low-attachment media was added after 7 days. Wells were imaged using 4x magnification on a computer-assisted phase contrast microscope (Nikon, Tokyo, Japan). Spheres larger than 100 micrometers were counted.

\section{Migration}

For migration cells were serum-starved overnight and scratch wounds were created using a sterile pipette tip on the cell monolayer or by plating cells in 2-well culture inserts (Ibidi, Madison, $\mathrm{WI}$ ). Cell migration rates were determined by measuring the distance between cell 
fronts after the indicated number of days in culture. The distance between the two edges at multiple points was quantified using ImageJ at the indicated timepoints.

\section{Co-treatment and interaction}

Cells were treated with doxorubicin (Selleckchem, Houston, TX, USA), paclitaxel (Selleckchem), OpA, 3-deoxy OpA, or matched-percentage DMSO in decreasing concentrations and incubated for 72 hours before measuring viability using MTS. Interactions were quantified using the Combenefit program with the Loewe model and dose-response surface mapping ${ }^{71}$.

\section{Tumor growth}

Female Scid/bg (CB17.Cg-PrkdcscidLystbg-J/Crl) mice (5-8 weeks old) were obtained from Charles River Laboratories (Wilmington, MA, USA). Animals were maintained under clean room conditions in sterile filter top cages with autoclaved bedding and housed on high efficiency particulate air-filtered ventilated racks. Animals received sterile rodent chow and acidified water ad libitum. All of the procedures were conducted in accordance with the Institute for Laboratory Animal Research Guide for the Care and Use of Laboratory Animals and with Baylor University Animal Care and Use Committee guidelines. HMLE-Ras + Twist cells were harvested, pelleted by centrifugation at 2000xg for 2 minutes, and resuspended in sterile serum-free medium supplemented with $30 \%$ to $50 \%$ Matrigel (BD Biosciences, San Jose, CA, USA). Cells ( $2 \times 10^{6}$ in 100 aliquots) were implanted into the left fourth mammary fat of each mouse and allowed to grow to the designated size, as measured by caliper, before the administration of OpA at $5 \mathrm{mg} / \mathrm{kg}$ or $10 \mathrm{mg} / \mathrm{kg}$ three times weekly for three weeks. Tumor volume and body weight were recorded concurrently to injection protocol ${ }^{72}$. At designated 
times, mice were humanely euthanized, and tumors were collected. Experiments were approved by Baylor University IACUC (\#1441130).

\section{Statistical analysis}

Unless otherwise stated, statistical differences were determined using a student's T-test. The GraphPad PRISM software v6 was used to perform these analyses. Statistical significance levels are annotated as n.s. = non-significant, ${ }^{\star} p<0.05,{ }^{* \star} p<0.01,{ }^{\star \star *} p<0.001,{ }^{* \star \star *} p<$ 0.0001 .

\section{Acknowledgements}

We acknowledge the entire Taube Lab for invaluable discussion and advice. Also, we appreciate the assistance of Dr. Igor Bado from Baylor College of Medicine for advice regarding Combenefit. Graphical abstract created with BioRender. The authors also thank Dr. Maurizio Vurro (Institute of Sciences and Food Production, CNR, Bari, Italy) for the supply of Drechslera gigantea culture filtrates. This work was supported by the Cancer Prevention and Research Institute of Texas, grant \#RP180771 to J.H.T. and D.R.

\section{Author Contributions}

Experiments performed by K.N.R. with contributions from S.S., P.S., S.P., and A.I. Isolation and characterization of ophiobolins by M.M., A.B., A.E., and A.K. Novel syntheses by Y.T. and D.R. Study design, manuscript drafting by K.N.R. and J.H.T., with editing by D.R. and A.K. All authors read and approved the final manuscript.

\section{Competing Interests}

The authors declare no competing interests. 
bioRxiv preprint doi: https://doi.org/10.1101/2020.05.06.079343; this version posted May 8, 2020. The copyright holder for this preprint (which was not certified by peer review) is the author/funder, who has granted bioRxiv a license to display the preprint in perpetuity. It is made available under aCC-BY-NC-ND 4.0 International license. 


\section{$\underline{\text { References }}$}

1 Carey, L. A. et al. The Triple Negative Paradox: Primary Tumor Chemosensitivity of Breast Cancer Subtypes. Clinical Cancer Research 13, 2329-2334, doi:10.1158/1078-0432.CCR-06-1109 (2007).

2 Cortazar, P. \& Geyer, C. E. Pathological Complete Response in Neoadjuvant Treatment of Breast Cancer. Annals of Surgical Oncology 22, 1441-1446, doi:10.1245/s10434-015-4404-8 (2015).

3 Foulkes, W. D., Smith, I. E. \& Reis-Filho, J. S. Triple-Negative Breast Cancer. New Engl J Med 363, 19381948, doi:10.1056/NEJMra1001389 (2010).

4 Haddad, T. C. \& Goetz, M. P. Landscape of Neoadjuvant Therapy for Breast Cancer. Annals of Surgical Oncology 22, 1408-1415, doi:10.1245/s10434-015-4405-7 (2015).

5 Liedtke, C. et al. Response to Neoadjuvant Therapy and Long-Term Survival in Patients With TripleNegative Breast Cancer. Journal of Clinical Oncology 26, 1275-1281, doi:10.1200/JCO.2007.14.4147 (2008).

6 Symmans, W. F. et al. Measurement of Residual Breast Cancer Burden to Predict Survival After Neoadjuvant Chemotherapy. Journal of Clinical Oncology 25, 4414-4422, doi:10.1200/JC0.2007.10.6823 (2007).

7 Chacón, R. D. \& Costanzo, M. V. Triple-negative breast cancer. Breast Cancer Res. 12 Suppl 2, S3, doi:10.1186/bcr2574 (2010).

8 Hudis, C. A. \& Gianni, L. Triple-negative breast cancer: an unmet medical need. Oncologist 16 Suppl 1, 111, doi:10.1634/theoncologist.2011-S1-01 (2011).

9 Mustacchi, G. \& De Laurentiis, M. The role of taxanes in triple-negative breast cancer: literature review. Drug Des Devel Ther 9, 4303-4318, doi:10.2147/DDDT.S86105 (2015).

$10 \mathrm{Wu}, \mathrm{J} ., \mathrm{Li}, \mathrm{S} ., \mathrm{Jia}, \mathrm{W} . \&$ Su, F. Response and prognosis of taxanes and anthracyclines neoadjuvant chemotherapy in patients with triple-negative breast cancer. J Cancer Res Clin Oncol 137, 1505, doi:10.1007/s00432-011-1029-6 (2011).

11 Dent, R. et al. Triple-Negative Breast Cancer: Clinical Features and Patterns of Recurrence. Clinical Cancer Research 13, 4429-4434, doi:10.1158/1078-0432.CCR-06-3045 (2007).

12 Hennessy, B. T. et al. Characterization of a naturally occurring breast cancer subset enriched in epithelial-to-mesenchymal transition and stem cell characteristics. Cancer research 69, 4116-4124, doi:10.1158/0008-5472.CAN-08-3441 (2009).

13 Prat, A. \& Perou, C. M. Deconstructing the molecular portraits of breast cancer. Molecular oncology 5, 523, doi:10.1016/j.molonc.2010.11.003 (2011).

14 Creighton, C. J. et al. Residual breast cancers after conventional therapy display mesenchymal as well as tumor-initiating features. Proceedings of the National Academy of Sciences 106, 13820-13825, doi:10.1073/pnas.0905718106 (2009).

15 Echeverria, G. V. et al. Resistance to neoadjuvant chemotherapy in triple-negative breast cancer mediated by a reversible drug-tolerant state. Sci. Transl. Med. 11, eaav0936, doi:10.1126/scitransImed.aav0936 (2019).

16 Mani, S. A. et al. The Epithelial-Mesenchymal Transition Generates Cells with Properties of Stem Cells. Cell 133, 704-715, doi:10.1016/j.cell.2008.03.027 (2008).

17 Lawson, D. A. et al. Single-cell analysis reveals a stem-cell program in human metastatic breast cancer cells. Nature 526, 131-+, doi:10.1038/nature15260 (2015).

18 Mani, S. A. et al. The epithelial-mesenchymal transition generates cells with properties of stem cells. Cell 133, 704-715, doi:10.1016/j.cell.2008.03.027 (2008).

19 Morel, A. P. et al. Generation of breast cancer stem cells through epithelial-mesenchymal transition. PloS one 3, e2888, doi:10.1371/journal.pone.0002888 (2008).

20 Burk, U. et al. A reciprocal repression between ZEB1 and members of the miR-200 family promotes EMT and invasion in cancer cells. EMBO reports 9, 582-589, doi:10.1038/embor.2008.74 (2008). 
Hollier, B. G. et al. FOXC2 Expression Links Epithelial-Mesenchymal Transition and Stem Cell Properties in Breast Cancer. Cancer research 73, 1981-1992, doi:10.1158/0008-5472.CAN-12-2962 (2013).

22 Korpal, M., Lee, E. S., Hu, G. \& Kang, Y. The miR-200 Family Inhibits Epithelial-Mesenchymal Transition and Cancer Cell Migration by Direct Targeting of E-cadherin Transcriptional Repressors ZEB1 and ZEB2. Journal of Biological Chemistry 283, 14910-14914, doi:10.1074/jbc.C800074200 (2008).

23 Mani, S. A. et al. Mesenchyme Forkhead 1 (FOXC2) plays a key role in metastasis and is associated with aggressive basal-like breast cancers. Proceedings of the National Academy of Sciences 104, 10069-10074, doi:10.1073/pnas.0703900104 (2007).

24 Park, S. M., Gaur, A. B., Lengyel, E. \& Peter, M. E. The miR-200 family determines the epithelial phenotype of cancer cells by targeting the E-cadherin repressors ZEB1 and ZEB2. Genes \& development 22, 894-907, doi:10.1101/gad.1640608 (2008).

25 Wellner, U. et al. The EMT-activator ZEB1 promotes tumorigenicity by repressing stemness-inhibiting microRNAs. Nature cell biology 11, 1487-1495, doi:10.1038/ncb1998 (2009).

26 Yang, J. et al. Twist, a Master Regulator of Morphogenesis, Plays an Essential Role in Tumor Metastasis. Cell 117, 927-939, doi:10.1016/j.cell.2004.06.006 (2004).

27 Bao, S. D. et al. Glioma stem cells promote radioresistance by preferential activation of the DNA damage response. Nature 444, 756-760, doi:10.1038/nature05236 (2006).

28 Hirschmann-Jax, C. et al. A distinct "side population" of cells with high drug efflux capacity in human tumor cells. Proceedings of the National Academy of Sciences 101, 14228-14233, doi:10.1073/pnas.0400067101 (2004).

29 Sharma, S. V. et al. A Chromatin-Mediated Reversible Drug-Tolerant State in Cancer Cell Subpopulations. Cell 141, 69-80, doi:10.1016/j.cell.2010.02.027 (2010).

30 Singh, A. \& Settleman, J. EMT, cancer stem cells and drug resistance: an emerging axis of evil in the war on cancer. Oncogene 29, 4741-4751, doi:10.1038/onc.2010.215 (2010).

31 Voulgari, A. \& Pintzas, A. Epithelial-mesenchymal transition in cancer metastasis: Mechanisms, markers and strategies to overcome drug resistance in the clinic. Biochimica et Biophysica Acta (BBA) - Reviews on Cancer 1796, 75-90, doi:10.1016/j.bbcan.2009.03.002 (2009).

32 Witta, S. E. et al. Restoring E-Cadherin Expression Increases Sensitivity to Epidermal Growth Factor Receptor Inhibitors in Lung Cancer Cell Lines. Cancer research 66, 944-950, doi:10.1158/0008-5472.CAN05-1988 (2006).

33 Naujokat, C. \& Steinhart, R. Salinomycin as a Drug for Targeting Human Cancer Stem Cells. BioMed Research International (2012).

$34 \mathrm{Li}, \mathrm{Y}$. \& Zhang, T. Targeting cancer stem cells by curcumin and clinical applications. Cancer letters 346 , 197-205, doi:10.1016/j.canlet.2014.01.012 (2014).

35 Liu, H.-T. \& Ho, Y.-S. Anticancer effect of curcumin on breast cancer and stem cells. Food Science and Human Wellness 7, 134-137, doi:10.1016/j.fshw.2018.06.001 (2018).

36 Takebe, N. et al. Targeting Notch, Hedgehog, and Wnt pathways in cancer stem cells: clinical update. Nature Reviews Clinical Oncology 12, 445-464, doi:10.1038/nrclinonc.2015.61 (2015).

37 Seo, H. S. et al. Quercetin induces caspase-dependent extrinsic apoptosis through inhibition of signal transducer and activator of transcription 3 signaling in HER2-overexpressing BT-474 breast cancer cells. Oncology reports 36, 31-42, doi:10.3892/or.2016.4786 (2016).

38 Masi, M., Dasari, R., Evidente, A., Mathieu, V. \& Kornienko, A. Chemistry and biology of ophiobolin A and its congeners. Bioorg Med Chem Lett 29, 859-869, doi:10.1016/j.bmcl.2019.02.007 (2019).

39 Bencsik, O. et al. Ophiobolin A from Bipolaris oryzae Perturbs Motility and Membrane Integrities of Porcine Sperm and Induces Cell Death on Mammalian Somatic Cell Lines. Toxins 6, 2857-2871, doi:10.3390/toxins6092857 (2014).

40 Bury, M. et al. Ophiobolin A induces paraptosis-like cell death in human glioblastoma cells by decreasing BKCa channel activity. Cell death \& disease 4, doi:ARTN e56110.1038/cddis.2013.85 (2013). 
Cocucci, S. M., Morgutti, S., Cocucci, M. \& Gianani, L. Effects of ophiobolin A on potassium permeability, transmembrane electrical potential and proton extrusion in maize roots. Plant Science Letters 32, 9-16, doi:10.1016/0304-4211(83)90093-7 (1983).

42 Kim, I. Y. et al. Ophiobolin A kills human glioblastoma cells by inducing endoplasmic reticulum stress via disruption of thiol proteostasis. Oncotarget 8, doi:10.18632/oncotarget.22537 (2017).

43 Rodolfo, C. et al. Ophiobolin A Induces Autophagy and Activates the Mitochondrial Pathway of Apoptosis in Human Melanoma Cells. PloS one 11, e0167672, doi:10.1371/journal.pone.0167672 (2016). Pósa, A. et al. The effect of acute ophiobolin A treatment on HO-mediated inflammatory processes. Human \& Experimental Toxicology 36, 594-602, doi:10.1177/0960327116658107 (2017).

45 Najumudeen, A. K. et al. Cancer stem cell drugs target K-ras signaling in a stemness context. Oncogene 35, 5248-5262, doi:10.1038/onc.2016.59 (2016).

$46 \mathrm{Au}$, T. K., Chick, W. S. H. \& Leung, P. C. The biology of ophiobolins. Life Sciences 67, 733-742, doi:10.1016/S0024-3205(00)00668-8 (2000).

47 Dasari, R. et al. Fungal metabolite ophiobolin A as a promising anti-glioma agent: In vivo evaluation, structure-activity relationship and unique pyrrolylation of primary amines. Bioorg Med Chem Lett 25, 4544-4548, doi:10.1016/j.bmcl.2015.08.066 (2015). Malouf, G. G. et al. Architecture of epigenetic reprogramming following Twist1-mediated epithelialmesenchymal transition. Genome biology 14, R144, doi:10.1186/gb-2013-14-12-r144 (2013). Gupta, P. B. et al. Identification of Selective Inhibitors of Cancer Stem Cells by High-Throughput Screening. Cell 138, 645-659, doi:10.1016/j.cell.2009.06.034 (2009).

50 Yoon, M. J., Kim, E. H., Lim, J. H., Kwon, T. K. \& Choi, K. S. Superoxide anion and proteasomal dysfunction contribute to curcumin-induced paraptosis of malignant breast cancer cells. Free Radical Biology and Medicine 48, 713-726, doi:10.1016/j.freeradbiomed.2009.12.016 (2010).

51 Fan, P. et al. Genistein decreases the breast cancer stem-like cell population through Hedgehog pathway. Stem Cell Research \& Therapy 4, 146, doi:10.1186/scrt357 (2013).

52 Hothi, P. et al. High-Throughput Chemical Screens Identify Disulfiram as an Inhibitor of Human Glioblastoma Stem Cells. Oncotarget 3, doi:10.18632/oncotarget.707 (2012).

53 Cano, A. et al. The transcription factor snail controls epithelial-mesenchymal transitions by repressing Ecadherin expression. Nature cell biology 2, 76-83, doi:10.1038/35000025 (2000).

54 Shimono, Y. et al. Downregulation of miRNA-200c Links Breast Cancer Stem Cells with Normal Stem Cells. Cell 138, 592-603, doi:10.1016/j.cell.2009.07.011 (2009).

$55 \mathrm{Hu}, \mathrm{X}$. et al. Induction of cancer cell stemness by chemotherapy. Cell cycle 11, 2691-2698, doi:10.4161/cc.21021 (2012).

56 An, H. et al. Salinomycin Promotes Anoikis and Decreases the CD44+/CD24- Stem-Like Population via Inhibition of STAT3 Activation in MDA-MB-231 Cells. PloS one 10, e0141919, doi:10.1371/journal.pone.0141919 (2015).

57 Dewangan, J., Srivastava, S. \& Rath, S. K. Salinomycin: A new paradigm in cancer therapy. Tumor Biol 39, 101042831769503, doi:10.1177/1010428317695035 (2017).

58 Dominguez? ?omez, G. et al. Ivermectin as an inhibitor of cancer stem?like cells. Molecular medicine reports, doi:10.3892/mmr.2017.8231 (2017).

59 Kuo, S. Z. et al. Salinomycin induces cell death and differentiation in head and neck squamous cell carcinoma stem cells despite activation of epithelial-mesenchymal transition and Akt. BMC Cancer 12, 556, doi:10.1186/1471-2407-12-556 (2012).

60 Lu, Y. et al. Salinomycin exerts anticancer effects on human breast carcinoma MCF-7 cancer stem cells via modulation of Hedgehog signaling. Chemico-Biological Interactions 228, 100-107, doi:10.1016/j.cbi.2014.12.002 (2015). 
61 Zhou, Q. et al. Curcumin Improves the Tumoricidal Effect of Mitomycin C by Suppressing ABCG2 Expression in Stem Cell-Like Breast Cancer Cells. PloS one 10, e0136694, doi:10.1371/journal.pone.0136694 (2015).

62 Newman, D. J. \& Cragg, G. M. Natural Products as Sources of New Drugs from 1981 to 2014. J Nat Prod 79, 629-661, doi:10.1021/acs.jnatprod.5b01055 (2016).

63 Bhatia, D. R. et al. Anticancer activity of Ophiobolin A, isolated from the endophytic fungus Bipolaris setariae. Nat Prod Res 30, 1455-1458, doi:10.1080/14786419.2015.1062760 (2016).

64 Chidley, C., Trauger, S. A., Birsoy, K. \& O'Shea, E. K. The anticancer natural product ophiobolin A induces cytotoxicity by covalent modification of phosphatidylethanolamine. elife 5, e14601, doi:10.7554/eLife.14601 (2016).

65 Choi, B.-K. et al. New Ophiobolin Derivatives from the Marine Fungus Aspergillus flocculosus and Their Cytotoxicities against Cancer Cells. Mar Drugs 17, 346, doi:10.3390/md17060346 (2019).

66 Tian, W., Deng, Z. X. \& Hong, K. The Biological Activities of Sesterterpenoid-Type Ophiobolins. Mar Drugs 15, doi:ARTN 22910.3390/md15070229 (2017).

67 Elenbaas, B. et al. Human breast cancer cells generated by oncogenic transformation of primary mammary epithelial cells. Genes \& development 15, 50-65 (2001).

68 Evidente, A. et al. Herbicidal potential of ophiobolins produced by Drechslera gigantea. J Agric Food Chem 54, 1779-1783, doi:10.1021/jf052843I (2006).

69 Sugawara, F. et al. Phytotoxins from the pathogenic fungi Drechslera maydis and Drechslera sorghicola. Proceedings of the National Academy of Sciences of the United States of America 84, 3081-3085, doi:10.1073/pnas.84.10.3081 (1987).

70 Sugawara, F. et al. Some new phytotoxic ophiobolins produced by Drechslera oryzae. The Journal of Organic Chemistry 53, 2170-2172, doi:10.1021/jo00245a008 (1988).

71 Di Veroli, G. Y. et al. Combenefit: an interactive platform for the analysis and visualization of drug combinations. Bioinformatics 32, 2866-2868, doi:10.1093/bioinformatics/btw230 (2016).

72 Faustino-Rocha, A. et al. Estimation of rat mammary tumor volume using caliper and ultrasonography measurements. Lab Anim (NY) 42, 217-224, doi:10.1038/laban.254 (2013). 


\section{Figure Legends}

Figure 1: Sensitivity to OpA is enhanced by EMT. (A) Representative morphology of nontransformed, immortalized, mammary epithelial cells expressing Twist or control vector. Scale bar represents $20 \mu \mathrm{m}$. (B) Representative western blot showing E-cadherin and vimentin protein expression. Images cropped to show relevant bands. Full-length blots available in supplemental information. (C) Cytotoxic activity of the indicated compounds was measured, in triplicate, by MTS assay. Mean and standard deviation of IC50 values are reported. Selectivity index is calculated as (HMLE Vector IC50) / (HMLE Twist IC50). (D) Representative data indicating cytotoxicity as for the indicated cell lines. Error bars represent standard deviation. (E/F) Mean and standard deviation of IC50 values for OpA $(E), n=3$ or 4 , unpaired t-test and staurosporine $(F), n=2$, unpaired t-test. $n . d .=$ not determined, n.a. $=$ not applicable.

Figure 2: The EMT state is necessary for sensitivity to Ophiobolin. (A) Mean and standard deviation of miR-200c expression in HMLE Twist cells expressing ectopic miR-200c or a control vector. $\mathrm{N}=3$ (B) Mean and standard deviation of relative viability for indicated doses of OpA in HMLE and HMLE Twist cells expressing ectopic miR-200c or a control vector. $n=3$, unpaired t-test.

Figure 3: Treatment with OpA, but not an inactive congener, suppresses EMT-driven cell behavior. (A) Mean and standard deviation of relative viability for indicated doses of OpA in MCF7 and MDA-MB-231 cells, $n=8$, unpaired t-test. (B) Representative data indicating cytotoxicity of MDA-MB-231 to the indicated compounds. Black arrow indicates dose used for sub-cytotoxic dosing. (C) Representative morphology of MDA-MB-231 cells were treated with the indicated compounds at 30nM for 30 days. Scale bar $=100 \mu \mathrm{m}$. (D/E) MDA-MB-231 cells, treated with the indicated compounds at $30 \mathrm{nM}$ for 4 days, were cultured in clean media for 12 
hours then subjected to a wound healing assay. (F) MDA-MB-231 cells, treated with the indicated compounds at $30 \mathrm{nM}$ for 10 days, were cultured in clean media for 48 hours then subjected to a sphere-forming assay. $n=8$, unpaired t-test

Figure 4: Combinatorial activity for OpA with doxorubicin and paclitaxel. (A/B)

Representative data indicating cytotoxicity of MDA-MB-231 to a range of doses for OpA and doxorubicin (A) or OpA and paclitaxel (B). (C/D) Data from (A/B) are represented using Combenefit. Blue-shaded areas represent dose combinations with synergistic effects (E/F) Representative data indicating cytotoxicity of MDA-MB-231 to a range of doses for 3-deoxyOpA and doxorubicin (E) or 3-deoxy-OpA and paclitaxel (F).

\section{Figure 5: OpA is tolerated in vivo and suppresses tumor growth from cells over-} expressing Twist. (A/B) Mice, bearing tumors composed of HMLE-Ras Twist cell, were injected with $10 \mathrm{mg} / \mathrm{kg}(\mathrm{n}=2), 5 \mathrm{mg} / \mathrm{kg}(\mathrm{n}=5), 2.5 \mathrm{mg} / \mathrm{kg}(\mathrm{n}=3)$, of OpA, or vehicle control $(\mathrm{n}=8)$, thrice weekly for three weeks. (A) Body weight was tracked. Arrows indicate endpoint criterion met for an individual animal. Statistical significance measured using the Holm-Sidak method with an alpha of 5\%. (B) End-point tumor volume was compared via unpaired t-test, $n=4$. 
Figure 1- Sensitivity to OpA is enhanced by EMT

A

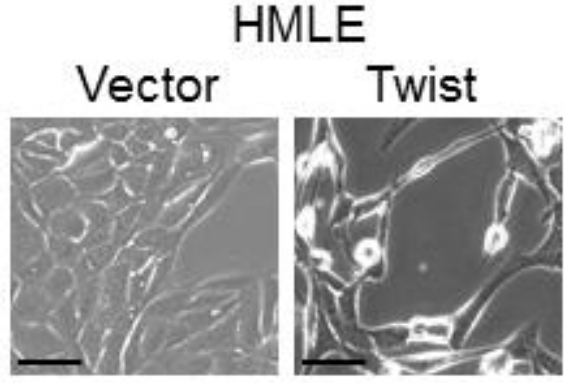

B

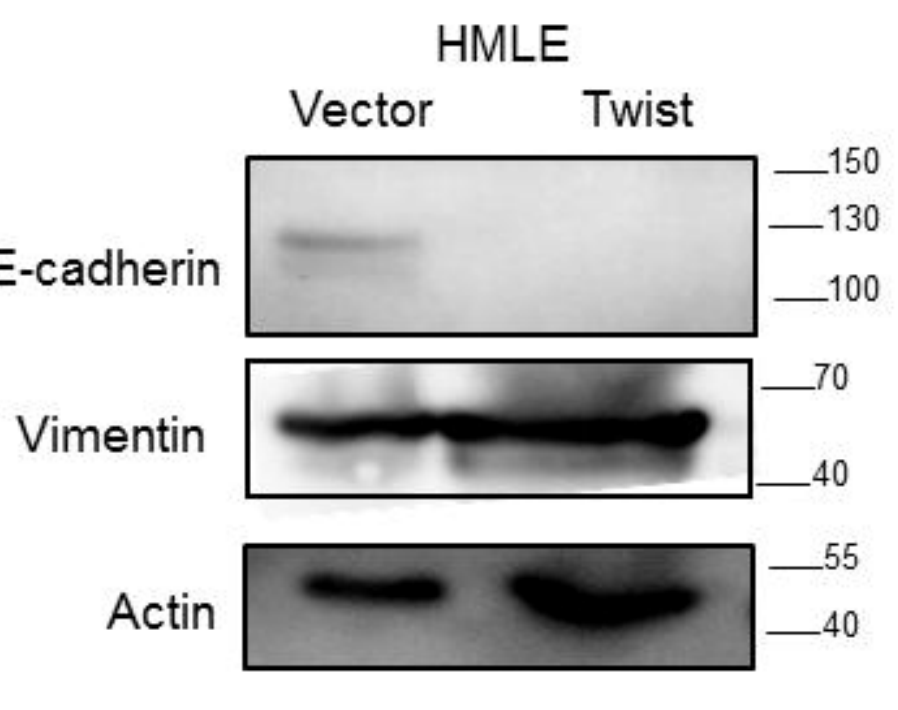

D

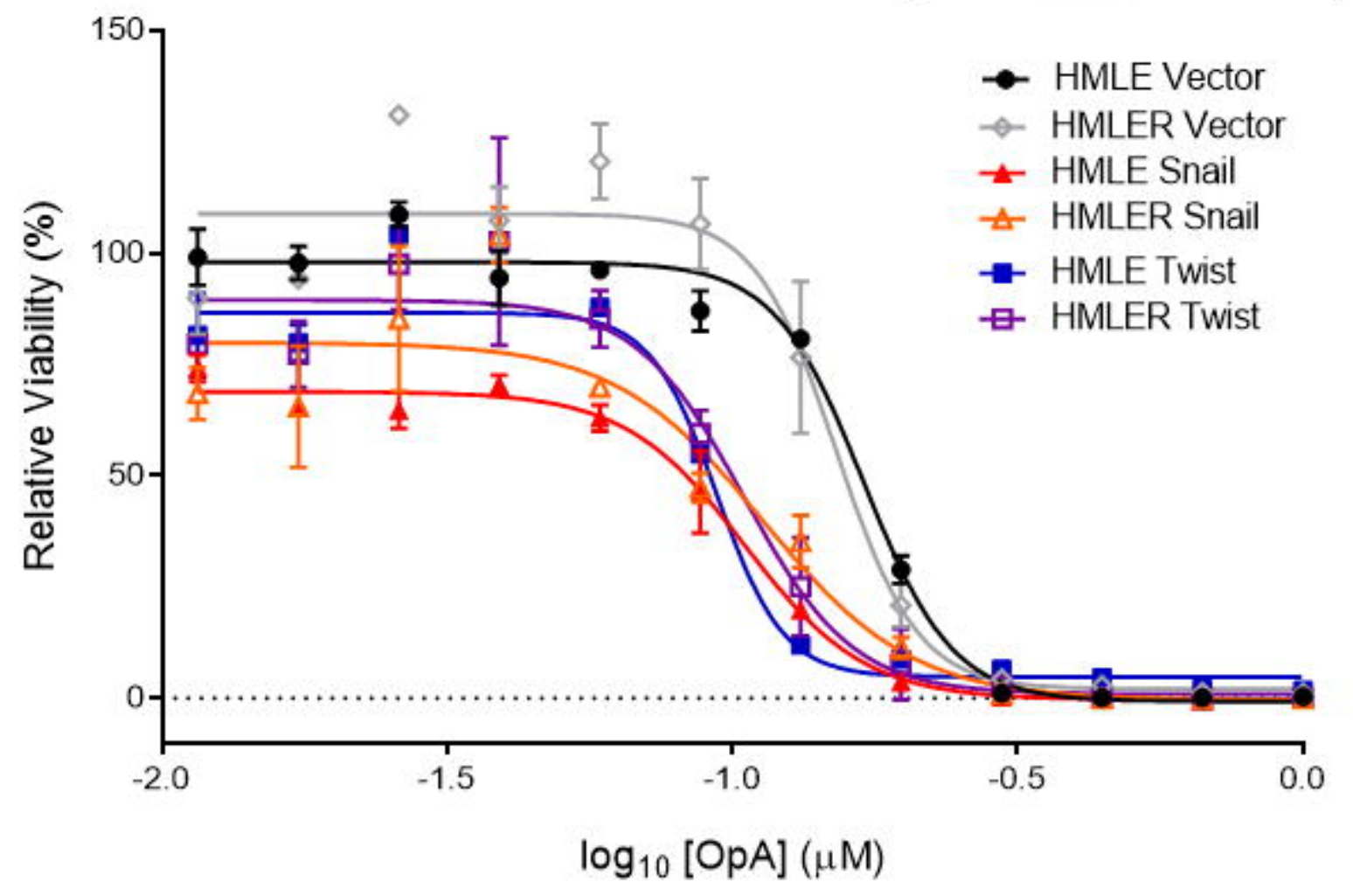

HMLER

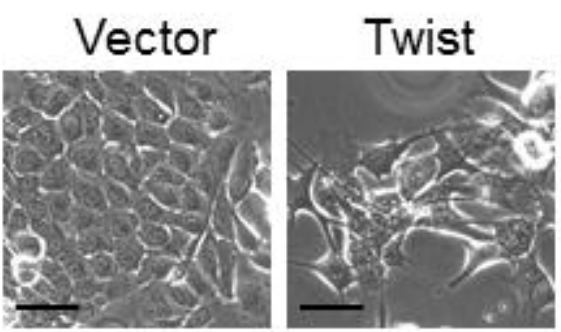

HMLER

Vector Twist

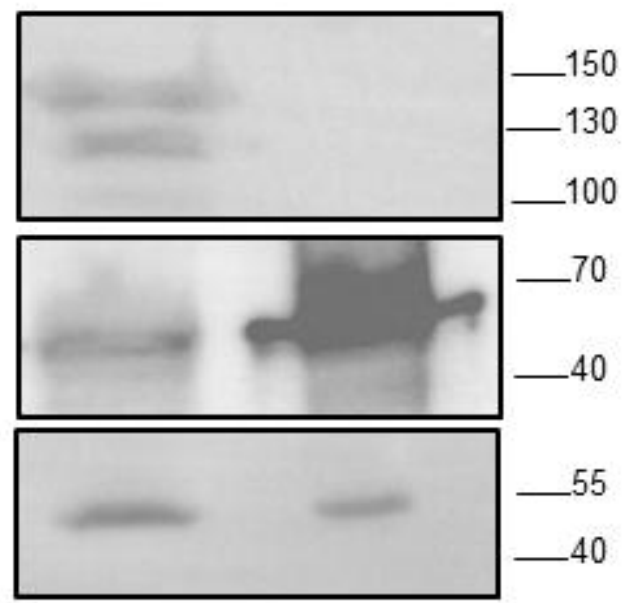

E

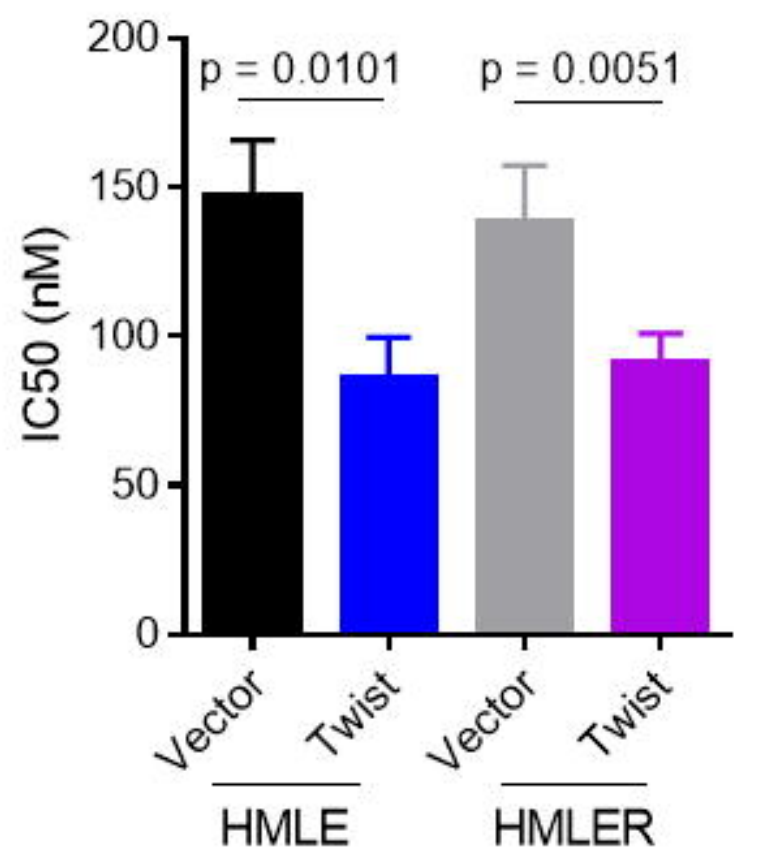

HMLE HMLER
$\mathrm{F}$

\section{5 nM Staurosporine}

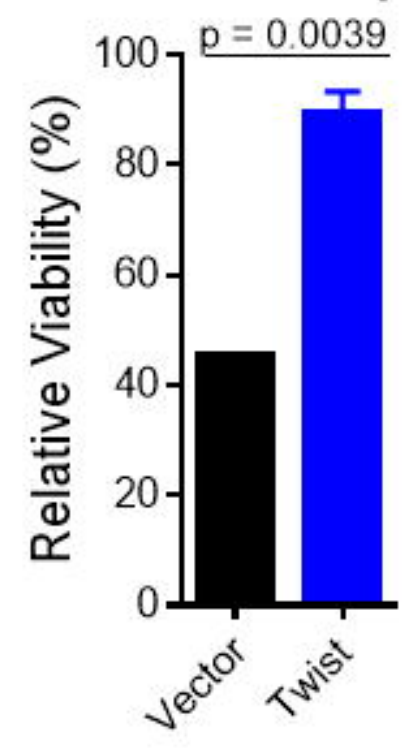

Selectivity Index

\begin{tabular}{|l|c|c|c|c|c|}
\hline \multirow{2}{*}{} & \multicolumn{4}{|c|}{ HMLER } & \multirow{2}{*}{$\begin{array}{c}\text { Selectivity } \\
\text { Index }\end{array}$} \\
\cline { 2 - 5 } IC50 $(\mu \mathrm{M})$ & \multicolumn{2}{|c|}{ Vector } & \multicolumn{2}{c|}{ Twist } & \\
\cline { 2 - 5 } & Mean & S.D. $(+/-)$ & Mean & S.D. $(+/-)$ & \\
\hline Salinomycin & 2.76 & 1.19 & 0.709 & 0.22 & 3.9 \\
\hline Ophiobolin A & 0.138 & 0.01 & 0.091 & 0.005 & 1.5 \\
\hline Curcumin & 0.075 & 0.009 & 0.099 & 0.02 & 0.8 \\
\hline Genistein & 0.011 & 0.003 & 0.018 & 0.003 & 0.6 \\
\hline Disulfiram & $>10.0$ & n.d. & $>10.0$ & n.d. & n.a. \\
\hline
\end{tabular}


Figure 2-The EMT state is necessary for sensitivity to Ophiobolin

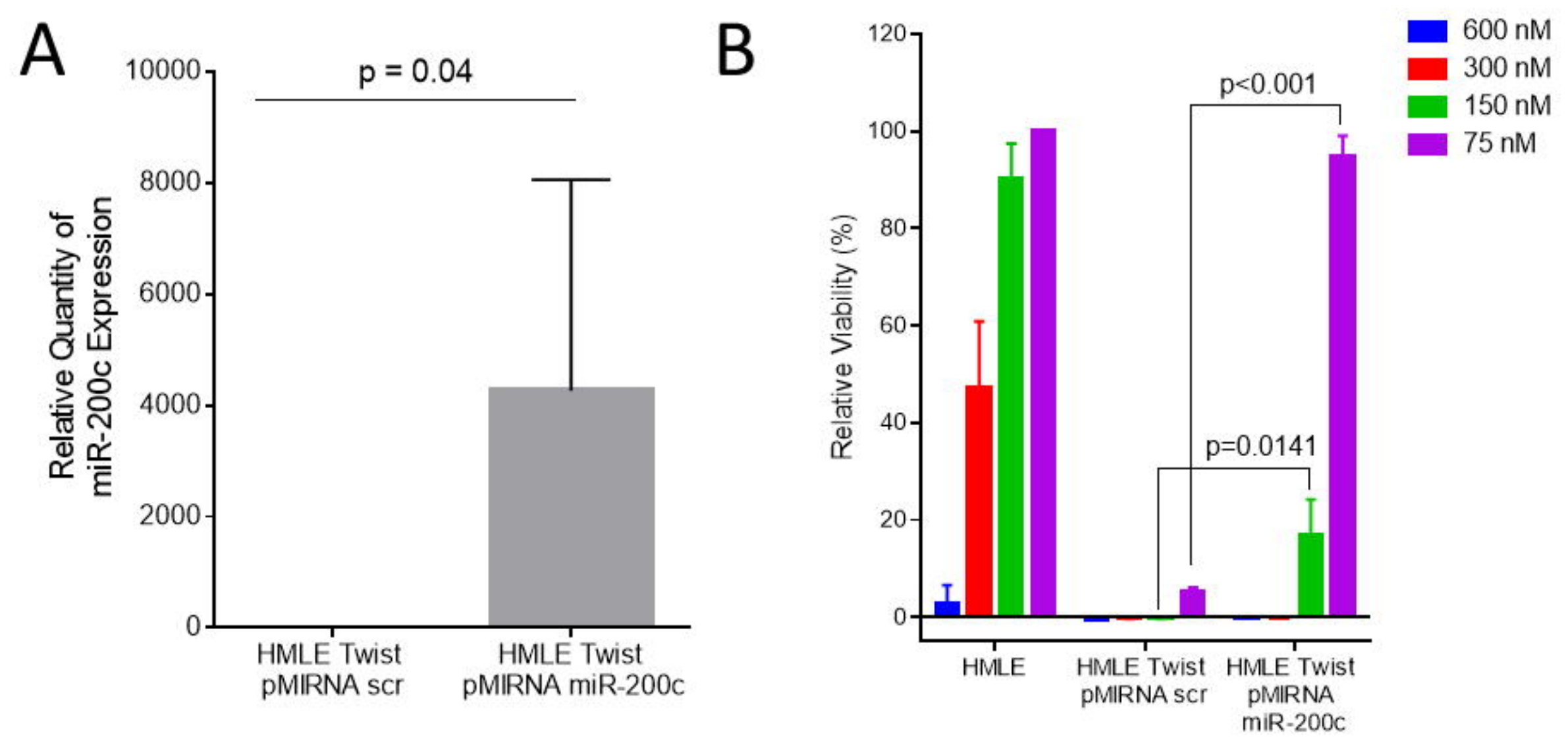


Figure 3-Treatment with OpA, but not an inactive congener, suppresses EMT-driven cell behavior

A

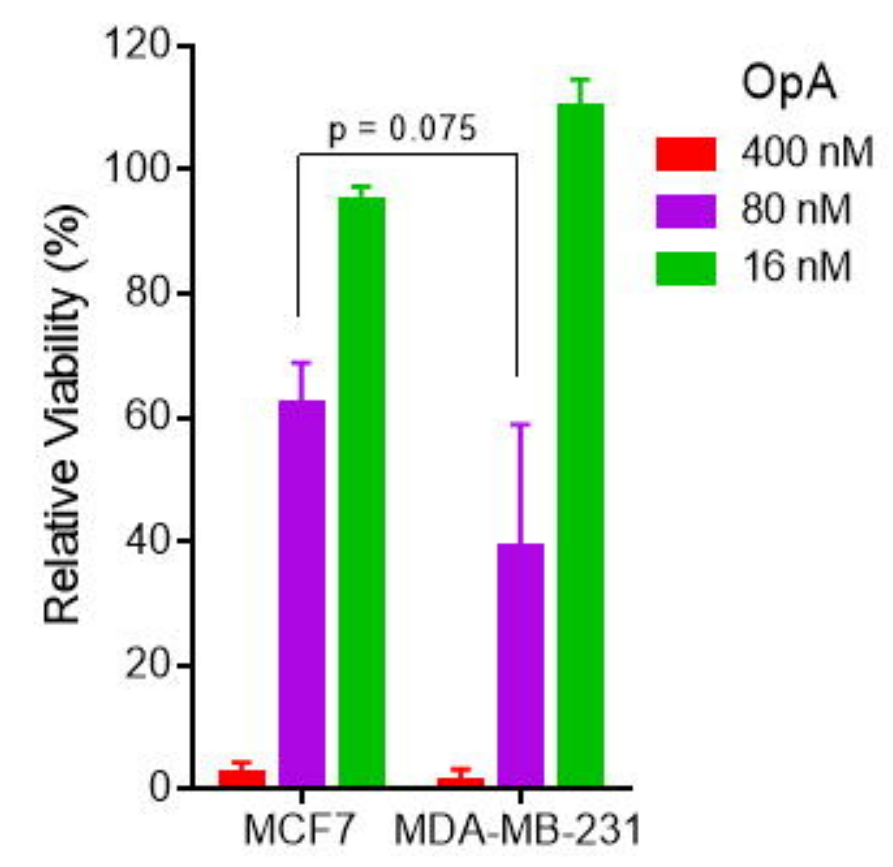

D

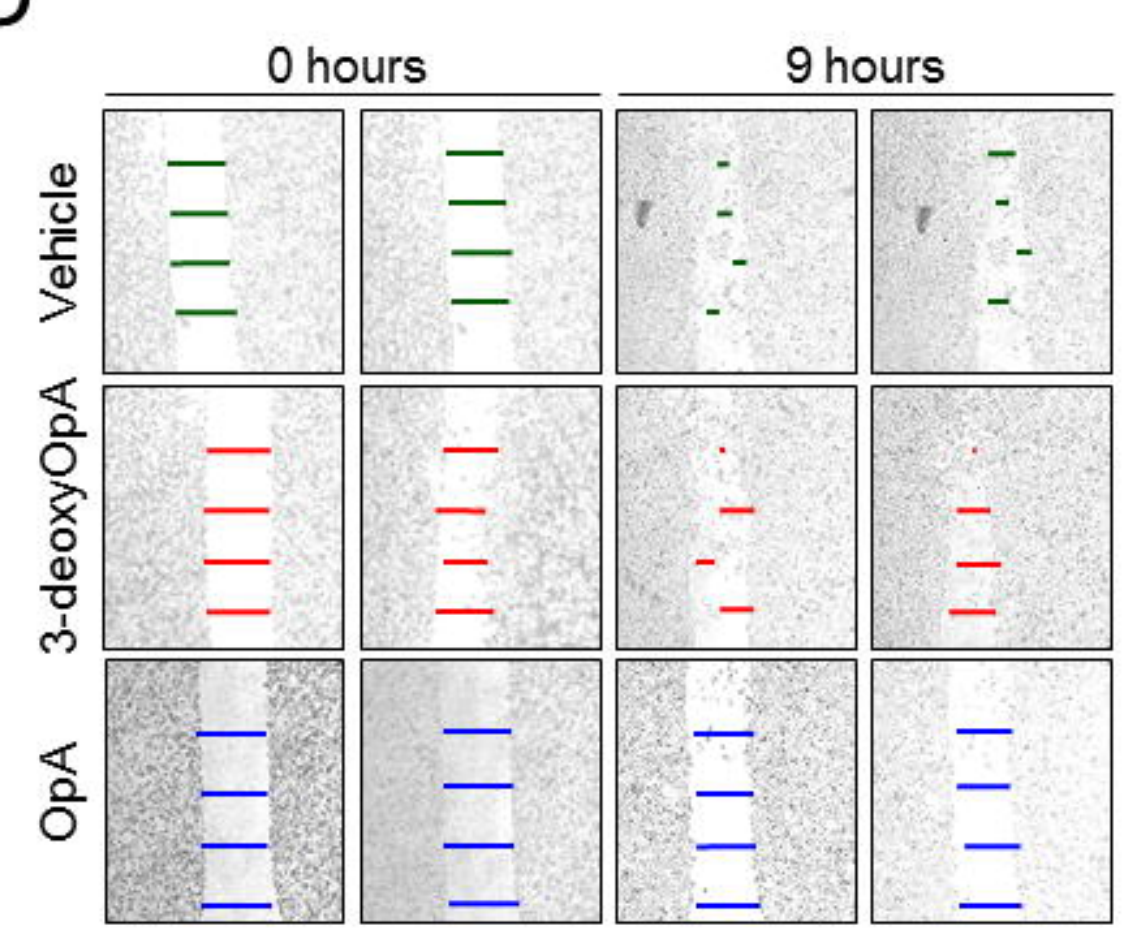

B

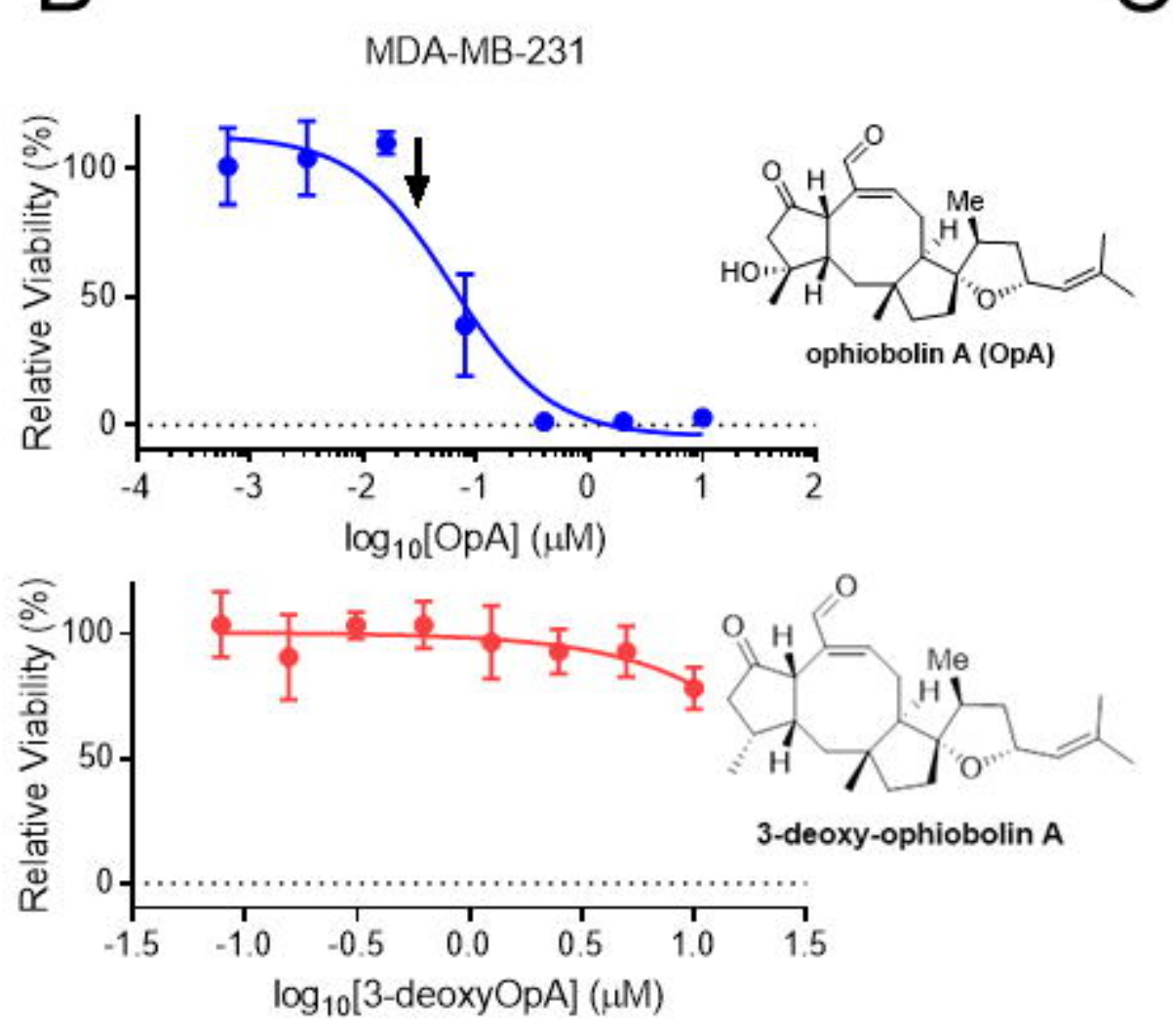

E

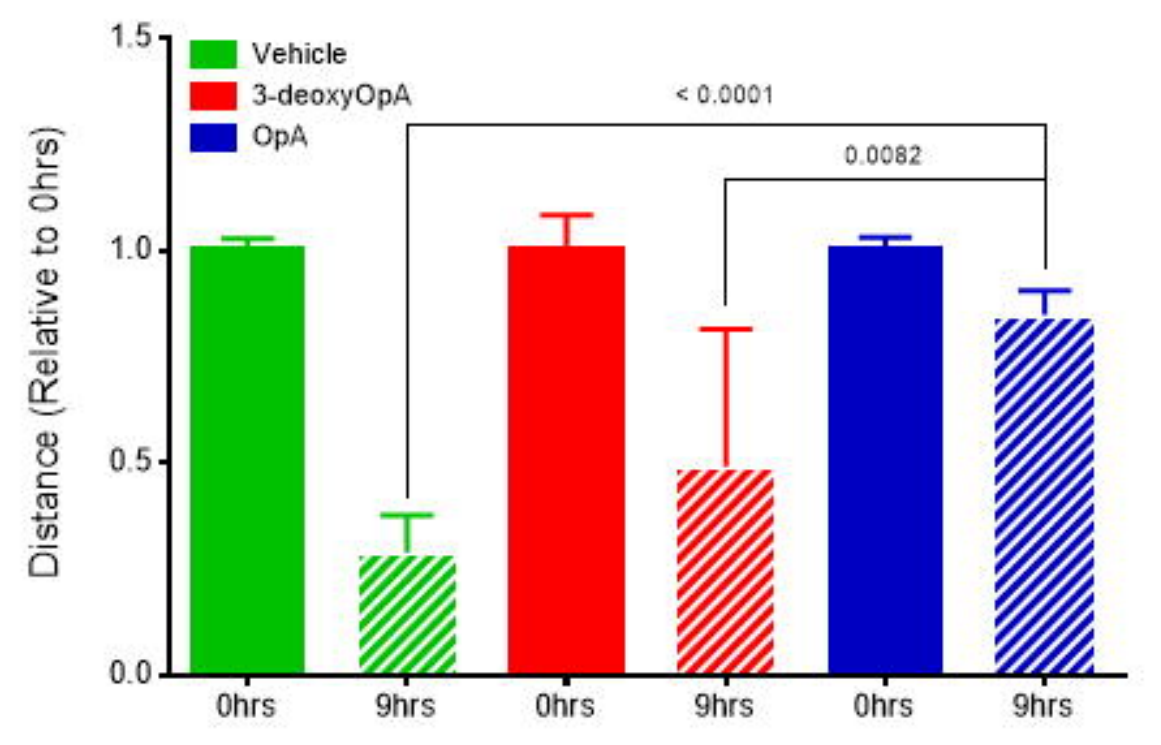

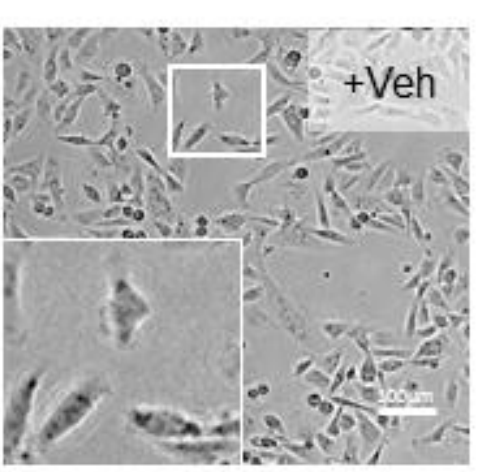

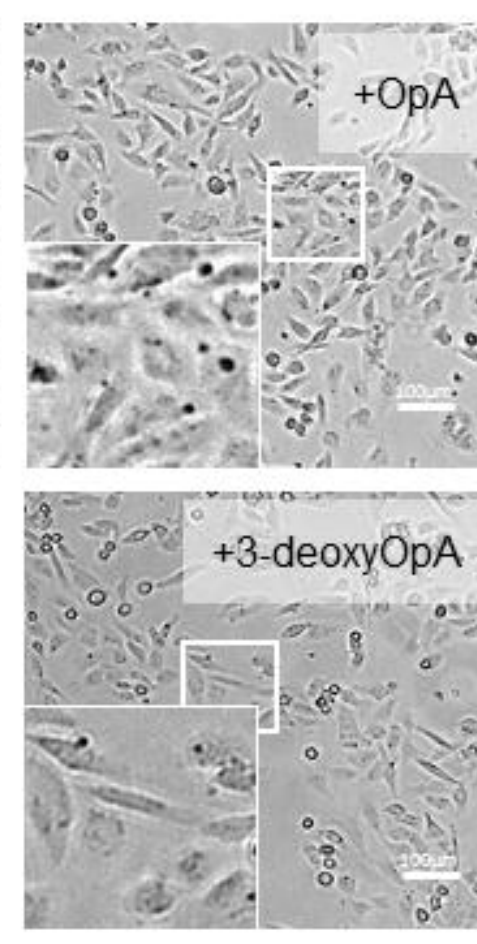

$\mathrm{F}$

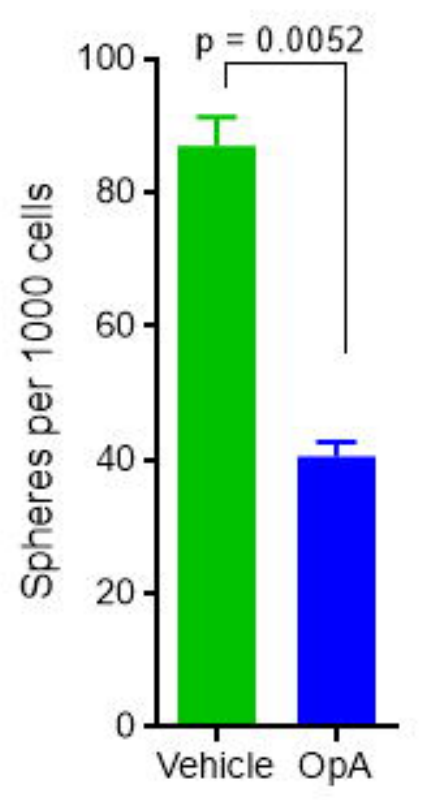


Figure 4-Combinatorial activity for OpA with doxorubicin and paclitaxel

A

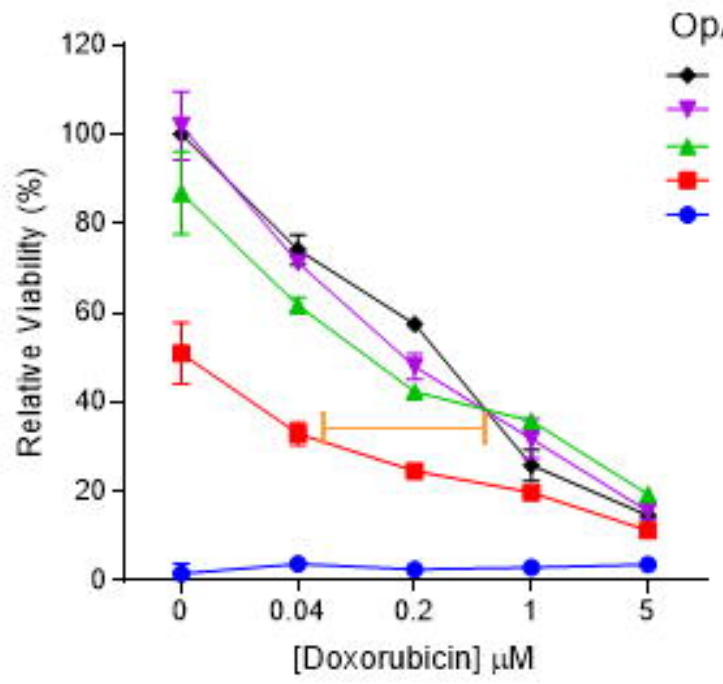

C

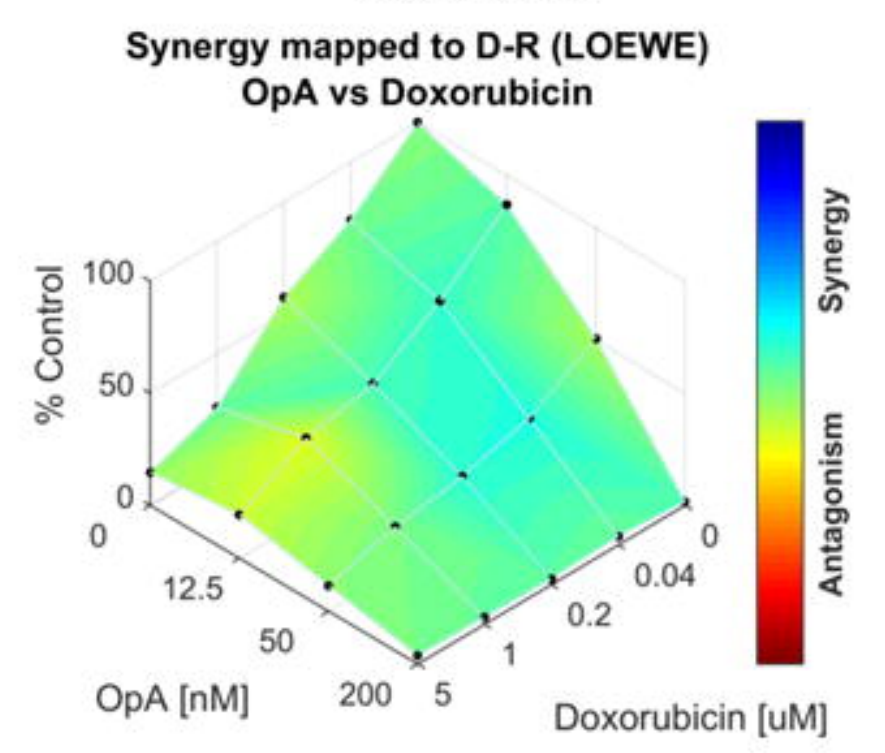

D

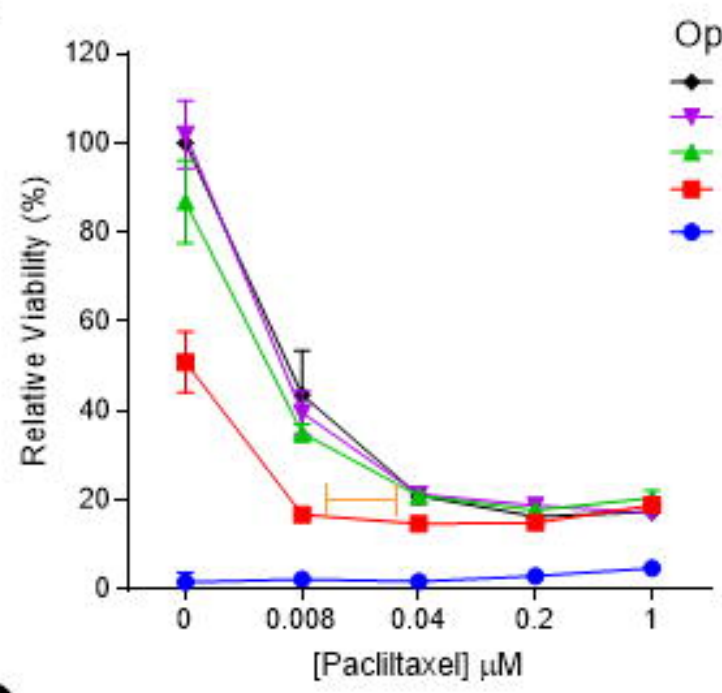

D Synergy mapped to D-R (LOEWE)

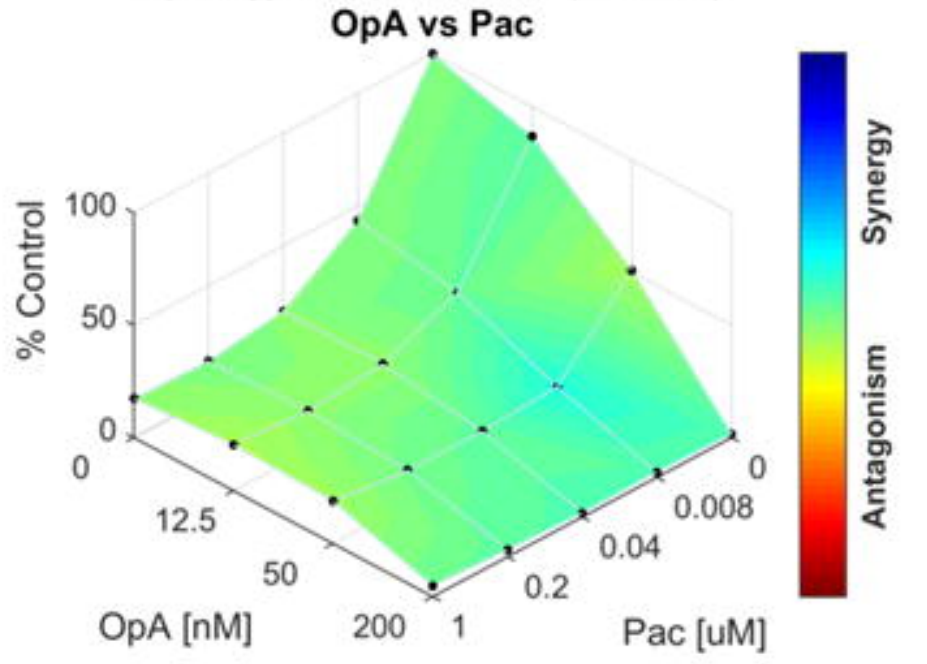

E
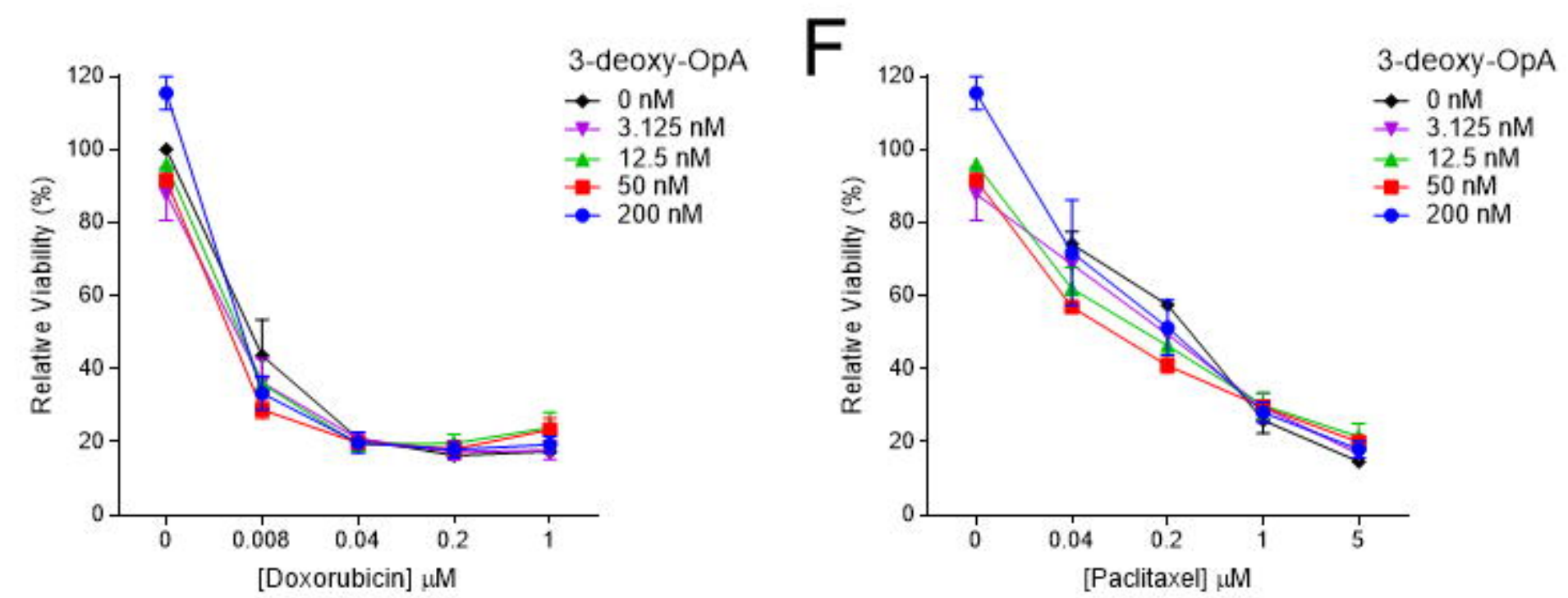
Figure 5-OpA is tolerated in vivo and suppresses tumor growth from cells over-expressing Twist.

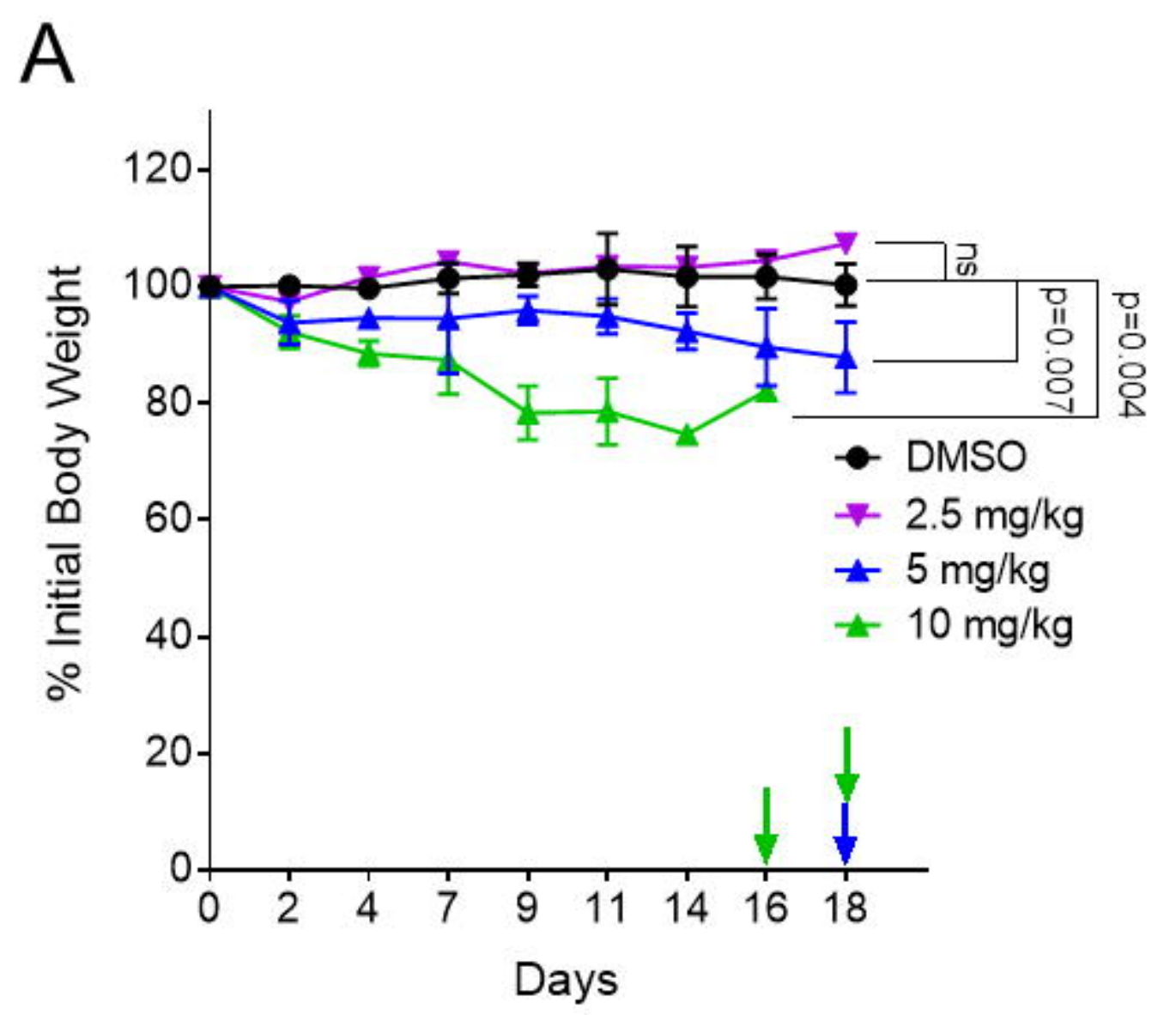

B

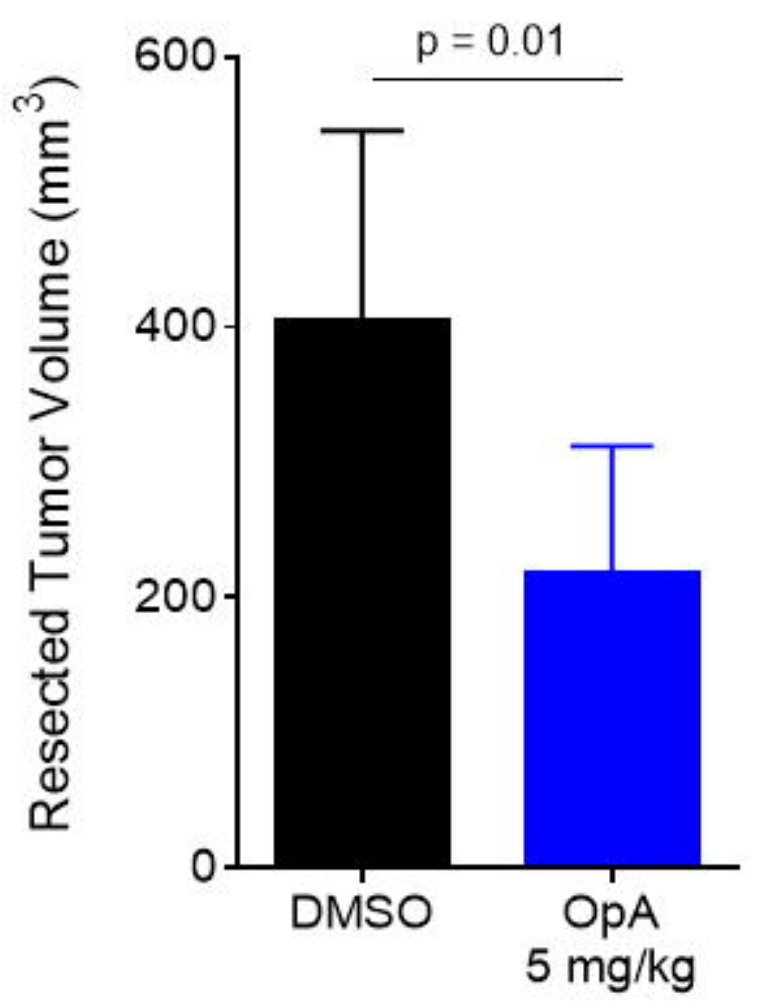

